\title{
Identification of significant immune-related genes for epilepsy via bioinformatics analysis
}

\author{
Xiaodan Luo, Tao Xiang, Hongmi Huang, Lin Ye, Yifei Huang, Yuan Wu \\ Department of Neurology, The First Affiliated Hospital of Guangxi Medical University, Nanning, China \\ Contributions: (I) Conception and design: X Luo, T Xiang; (II) Administrative support: H Huang, L Ye; (III) Provision of study materials or patients: \\ X Luo, T Xiang; (IV) Collection and assembly of data: X Luo, T Xiang; (V) Data analysis and interpretation: Y Wu; (VI) Manuscript writing: All \\ authors; (VII) Final approval of manuscript: All authors. \\ Correspondence to: Yuan Wu. Department of Neurology, The First Affiliated Hospital of Guangxi Medical University, 6 Shuangyong Road, Nanning \\ 530000, China. Email: wuyuan90@126.com.
}

Background: Epilepsy is one of the most common neurological disorders, but its underlying mechanism has remained obscure, and the role of immune-related genes (IRGs) in epilepsy have not yet been investigated. Therefore, in this study, we explored the association between IRGs and epilepsy.

Methods: An IRG list was collected from the ImmPort database. The gene expression profiles of GSE143272 were collected from the Gene Expression Omnibus (GEO) database (https://www.ncbi.nlm. nih.gov/geo/). Differentially expressed genes (DEGs) between epilepsy and normal samples were analyzed, and the intersections between IRGs and DEGs were identified using the VennDiagram package, with the intersected genes subjected to further analysis. Enrichment function for intersected genes were performed, constructed a protein-protein interaction (PPI) network via the Search Tool for the Retrieval of Interacting Genes/Proteins (STRING) database, and the hub genes (top 10) of the PPI network were calculated by the cytoHubba plug-in in Cytoscape. The top correlated genes were selected to perform correlation analysis with immune cells infiltration and expression levels. Finally, we performed validation of the top correlated genes transcriptional expression levels using an animal model.

Results: There were a total of 245 DEGs detected in GSE143272, among which 143 were upregulated and 102 downregulated genes in epilepsy. A total of 44 differential IRGs were obtained via intersection of DEGs and IRGs. Enrichment function analysis of DEGs showed that they played a significant role in immune response. The gene CXCL1 was the most correlated with other differentially expressed IRGs via the PPI network. The results of immune cell infiltration analysis indicated that epilepsy patients had higher activated mast cells infiltration $(\mathrm{P}=0.021)$, but lower activated CD4 memory $\mathrm{T}$ cells $(\mathrm{P}=0.001)$, resting CD4 memory $\mathrm{T}$ cells $(\mathrm{P}=0.011)$, and gamma delta $\mathrm{T}$ cells $(\mathrm{P}=0.038)$ infiltration. It was revealed that CXCL1 and activated mast cells $(\mathrm{R}=0.25, \mathrm{P}=0.019)$ and neutrophils $(\mathrm{R}=0.3, \mathrm{P}=0.0043)$, and a negative correlation with $\mathrm{T}$ cells gamma delta $(\mathrm{R}=-0.25, \mathrm{P}=0.018)$. The levels of $\mathrm{CXCL1}$ expression were significantly lower in epilepsy patients than those in normal samples.

Conclusions: In this study, the results showed that IRGs such as CXCL1 have a significant influence on epilepsy via regulation of immune cells infiltration.

Keywords: Epilepsy; immune-related genes (IRGs); CXCL1

Submitted May 17, 2021. Accepted for publication Jul 22, 2021.

doi: $10.21037 /$ atm-21-2792

View this article at: https://dx.doi.org/10.21037/atm-21-2792 


\section{Introduction}

According to the World Health Organization (WHO), epilepsy is the one of the most common neurological disorders. It is characterized by emotional and cognitive dysfunction, and about 70 million people are affected by this neurological disorder worldwide (1). Despite the widespread use of antiepileptic drugs (AEDs), about onethird of epilepsy patients do not respond to AEDs (2). Therefore, it is an urgent need to develop novel safe and effective therapeutic regimens for epilepsy. It has been shown that AEDs stop seizures but do not influence the underlying pathology or inhibit the progression of neurological disorders (3). Further understanding of the underlying mechanisms of epilepsy may foster the development of novel treatment regimens that not only block, but also effect the progression, of seizures.

To date, increasing studies have provided significant evidence to confirm that inflammation plays an essential role in epilepsy $(4,5)$. Anti-inflammatory agents such as steroids and other drugs have been shown to be useful in the treatment of some drug-resistant epilepsy patients (6), supporting the theory that inflammation one of the pathogenic influences in epilepsy. Further, when seizures occur, it has been shown that proinflammatory agents coincidentally increase (7). Inflammation is always accompanied by changes in the immune system. Chronic brain inflammation has been detected in patients with Rasmussen's encephalitis, and several cell types such as microglia, astrocytes, endothelial cells of the blood-brain barrier (BBB), and immune cells are involved in this process (8). Several studies have shown that immune system is overly activated in some patients with seizure disorders, furthering the high incidence of seizures in autoimmune disease patients, and it has also been found that limbic encephalitis can act as cause of epilepsy $(9,10)$. These evidences strongly support that immune activation and inflammation play key roles in several types of epilepsy. On this basis, an immune-modulating and anti-inflammatory therapeutic regimen may be effective in treating some forms of epilepsy. Regrettably, the research has mainly focused on epilepsy immune cells and immunerelated genes (IRGs) have not yet been identified. Herein, it is a pressing need to identify the significant immune cells and IRGs that have essential roles in epilepsy. In the present study, we used bioinformatics to analyze the significant IRGs, and then explore the relationship between IRGs and immune cell infiltration in relation to epilepsy.

We present the following article in accordance with the ARRIVE reporting checklist (available at https://dx.doi. org/10.21037/atm-21-2792).

\section{Methods}

\section{Data collection}

The IRG list was collected from the ImmPort database (https://www.immport.org). The gene expression profiles of GSE143272 with 91 epilepsy samples (blood) and 51 normal samples (blood) were collected from the Gene Expression Omnibus (GEO) database (https://www.ncbi. nlm.nih.gov/geo/). The study was conducted in accordance with the Declaration of Helsinki (as revised in 2013).

\section{Difference analysis of IRGs in epilepsy}

The gene differences analysis was performed (epilepsy VS normal) via wilcox.test in $\mathrm{R}$ software (https://www.r-project. org/), and the filtering standards were set as log fold change $\mid \log \mathrm{FCl}>0.3$ and $\mathrm{P}$ value $<0.05$. The ggplot2 package in $\mathrm{R}$ software was used to plot the heatmap and volcano of differentially expressed genes (DEGs). The intersection of DEGs and IRGs was carried out using the VennDiagram package, and the differential IRGs were used for subsequent analysis.

\section{Functional enrichment analysis of immune-related differential genes}

The potential functional enrichment of immune-related differential genes in epilepsy was explored using the $\mathrm{R}$ software with the aid of the clusterProfiler and ggplot2 packages. A q value $<0.05$ was considered as significant enrichment functions.

\section{Construction of protein-protein interaction (PPI) network}

We used the differential immune related genes to construct a PPI network through the Search Tool for the Retrieval of Interacting Genes/Proteins (STRING) database, and the hub genes (top 10) of the PPI network were calculated using the cytoHubba plug-in in Cytoscape software (version 3.8.1; https://cytoscape.org).

\section{Analysis of immune cells infiltration in epilepsy and normal samples}

The CIBERSORT algorithm can characterize cell composition based on gene expression profiles, thus 
estimating the relative abundance of 22 immune cells. We used the CIBERSORT algorithm to calculate the abundance of immune cells in 91 epilepsy samples and 51 normal samples and explore the differential expression between them. Furthermore, we also investigated the correlation between the expression of the PPI network's hub gene and 22 immune cells.

\section{Pentylenetetrazole-induced chronic epilepsy model}

Male specific pathogen-free (SPF) Sprague Dawley (SD) rats were provided by the Experimental Animal Center of Guangxi Medical University. Animals were habituated for 7 days under controlled standard conditions of temperature, humidity and lighting conditions (12-12 light-dark schedule with light on $8 \mathrm{AM}$ ) before experiment. Animals had food and water freely.

A total of 40 rats (body weight $200-250 \mathrm{~g}$, aged $8-10$ weeks) were randomly divided into 2 groups: control group and chronic epilepsy group, with 20 rats in each group. If any rats died during the modeling process, they were replenished to the number of 20 in each group. According to the experimental method of Tchekalarova et al. (11), the rats were administered an intraperitoneal injection of $1 \%$ pentylenetetrazol (PTZ; Sigma-Aldrich, St. Louis, MO, USA) at a dose of $40 \mathrm{mg} / \mathrm{kg}$ for 28 days in succession at 8:00 am to 10:00 am every second day. Consecutive seizures of 3 times of grade IV or above were considered to be completely ignited (12). Following, PTZ was injected intraperitoneally once a day to maintain the ignition effect and establish the rat model of chronic epilepsy. Control group animals were intraperitoneally injected with normal saline once at the same time for 28 consecutive days. The dose of PTZ was calculated according to body weight before injection. Rats were carefully observed for $30 \mathrm{~min}$ after each administration of PTZ, and the seizure degree was recorded. Racine classification criteria were used as follows (13): (I) chew or facial movements; (II) nodding regularly; (III) unilateral or bilateral forelimb clonus; (IV) bilateral for limb clonus and rearing; and (V) generalized convulsions, falling and rearing. The minimum sample size of experimental animals was calculated by using statistical methods based on the pre-experimental data, so as to reduce the number of animals under the condition of ensuring the comparability of experimental data. A protocol was prepared before the study without registration. Experiments were performed under a project license (No.: 202101003) granted by The First Affiliated Hospital of Guangxi Medical University ethics board, in compliance with Chinese guidelines for the care and use of animals.

\section{$R N A$ extraction and reverse transcription polymerase chain reaction}

The RNA isolation agent NucleoZOL 740404.6 (MachereyNagel GnbH \& Co.KG., Düren, Germany) was used to extract Total RNA, which was then dissolved in $20 \mu \mathrm{L}$ RNase-free water.

Using spectrophotometry to examine the purity and concentration of the RNA extracts, RNA purity was confirmed using a cutoff value of 1.8-2.0 (Abs $260 \mathrm{~nm} / \mathrm{Abs} 280 \mathrm{~nm}$ ).

The PrimeScript ${ }^{\mathrm{TM}}$ RT Master Mix (RR036A, Takara Bio., Kusatsu, Shiga, Japan) was used to synthesize the circular DNA (cDNA). The reverse transcription reaction system, incorporating $4 \mu \mathrm{L} 5 \times$ PrimeScript RT Master Mix, $1,000 \mathrm{ng}$ extracted total RNA, and RNase-free distilled $\mathrm{H}_{2} \mathrm{O}$ was added to the final volume to $20 \mu \mathrm{L}$. The reverse transcription reaction conditions were as follows: $37^{\circ} \mathrm{C}$ for $15 \mathrm{~min}$ followed by $2 \mathrm{~min}$ at $85^{\circ} \mathrm{C}$. After the reaction, the cDNA was stored at $-20^{\circ} \mathrm{C}$.

A FastStart Universal SYBR Green Master (4913914001, RoX Health GmbH, Berlin, Germany) was used for realtime quantitative PCR. The reaction system was as follows: $5 \mu \mathrm{L} 2 \times$ SYBR Green Master mix, $1 \mu \mathrm{L}$ cDNA, $0.6 \mu \mathrm{L}$ primer (forward and reverse, $10 \mu \mathrm{M}$ ), and RNase-free double distilled (dd) $\mathrm{H}_{2} \mathrm{O}$ to reach the final volume of $10 \mu \mathrm{L}$. The primer sequences of CXCL1 and $\beta$-actin (14) were shown as follows:

CXCL1 Forward primer ACCCAAACCGAAGTCATAGCE Reverse primer: GGGACACCCTTTAGCATCTT $\beta$-actin Forward primer: ACCGCGAGAAGATGACCCAG Reverse primer: GGATAGCACAGCCTGGATAGCAA

A 7500 fast thermocycler was used to perform the RT-PCR, and the reaction conditions were as follows: $10 \mathrm{~min}$ at $95^{\circ} \mathrm{C}$, followed by 40 cycles of $15 \mathrm{~s}$ at $95^{\circ} \mathrm{C}$ and $1 \mathrm{~min}$ at $60^{\circ} \mathrm{C}$. Each gene was analyzed by RT-PCR in triplicate. The gene expression of CXCL1 was normalized to $\beta$-actin, and a $\triangle \triangle \mathrm{Ct}$ method was used for the quantification of target gene expression.

\section{Statistical analysis}

The differences were analyzed via the Wilcox test method, and the correlation was analyzed via Spearman's correlation. The RT-PCR data was analyzed as mean \pm standard deviation (mean \pm SD) and analyzed with SPSS 26.0 (SPSS Inc., USA). The $t$-test was used to analysis the difference 

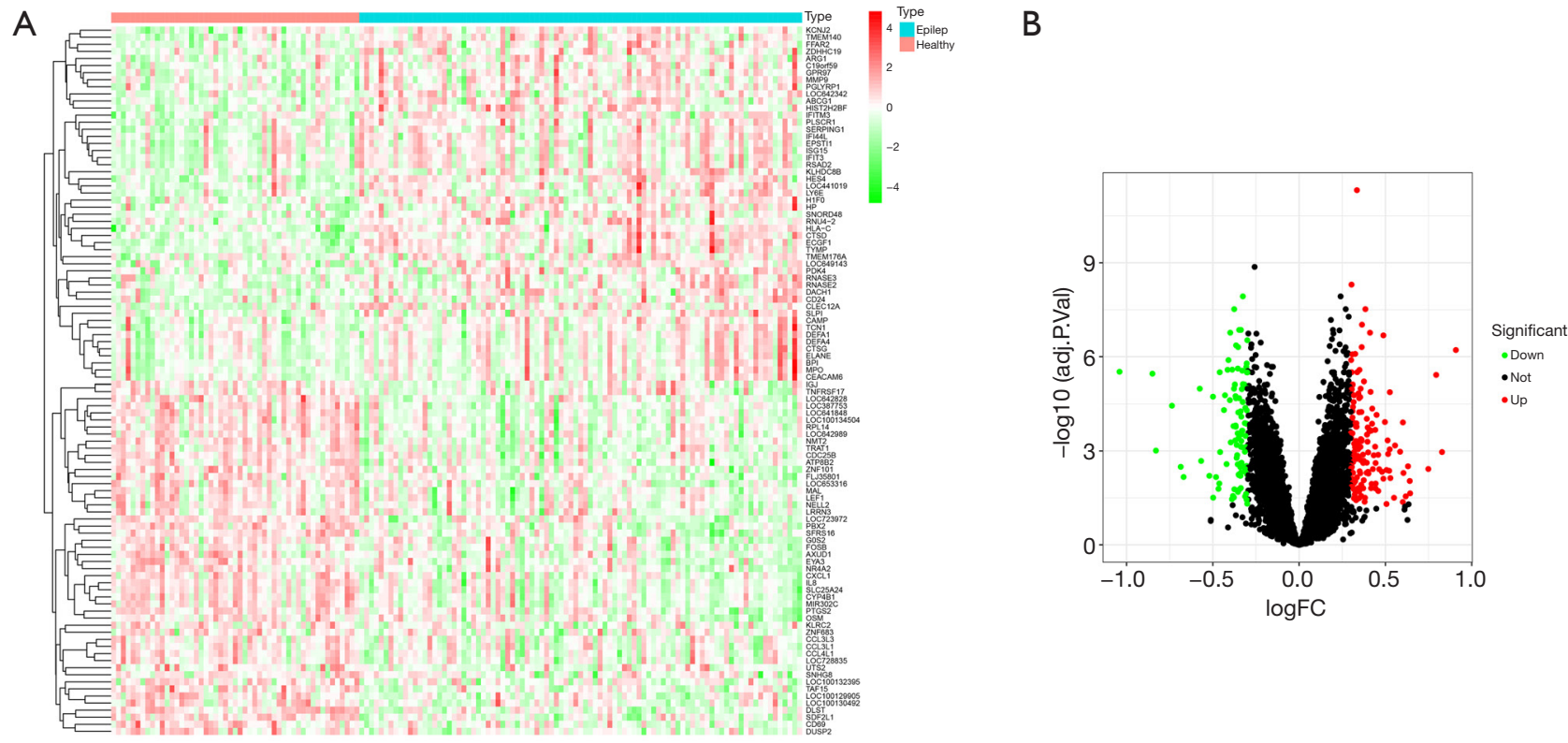

Figure 1 Extraction of immune-related DEGs in epilepsy. Heatmap plot (A) and Volcano (B) plot of DEGs. Green and red represent downregulated and up-regulated genes, respectively. DEGs, differentially expressed genes.

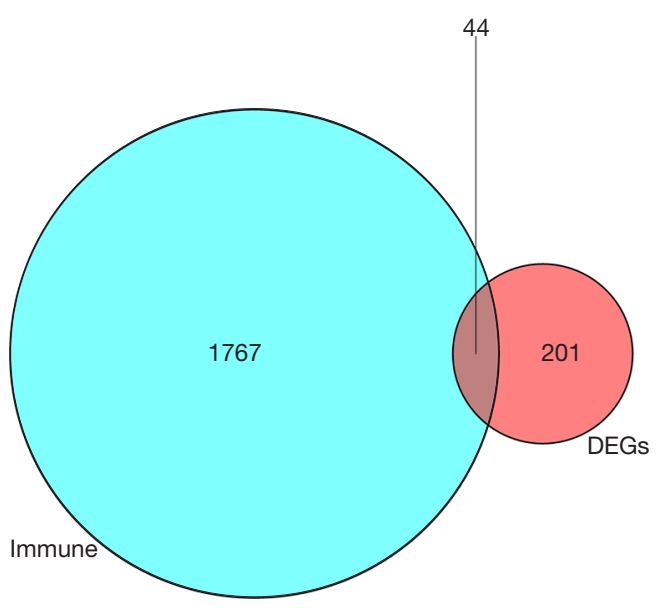

Figure 2 Intersecting genes of DEGs and IRGs. Red and cyan represent DEGs and immune related genes, respectively. DEGs, differentially expressed genes; IRGs, immune related genes.

between the control and chronic epilepsy groups. A P value $<0.05$ was considered statistically significant.

\section{Results}

\section{Extraction of immune-related DEGs in epilepsy}

There were a total of 245 DEGs in the GSE143272, among which there were 143 upregulated and 102 downregulated genes in epilepsy (Figure 1A,B). There were 1,811 IRGs in the ImmPort database, and 44 differential IRGs were obtained via intersection of DEGs and IRGs (Figure 2).

\section{Enrichment function analysis of immune related DEGs}

The results of immune related DEGs function analysis showed that the biological processes (BP) of these genes mainly participate in neutrophil degranulation, neutrophil activation involved in immune response, neutrophilmediated immunity, neutrophil activation, and defense response to bacterium. For cellular components (CC), DEGs may play the key role if secretory granule lumen and cytoplasmic vesicle lumen. According to molecular function (MF), immune related DEGs have a critical role in chemokine receptor binding, chemokine activity, major histocompatibility complex (MHC) protein complex binding, and so on (Figure $3 A, B)$. The pathway of immune related DEGs analysis showed that they are parts of several important pathways such as the NF-kappa B signaling pathway, viral protein interaction with cytokine and cytokine receptor, human cytomegalovirus infection, programmed death-ligand 1 (PD-L1) expression and PD-1 checkpoint pathway in cancer, antigen processing and presentation, and cytokine-cytokine receptor interaction 
A

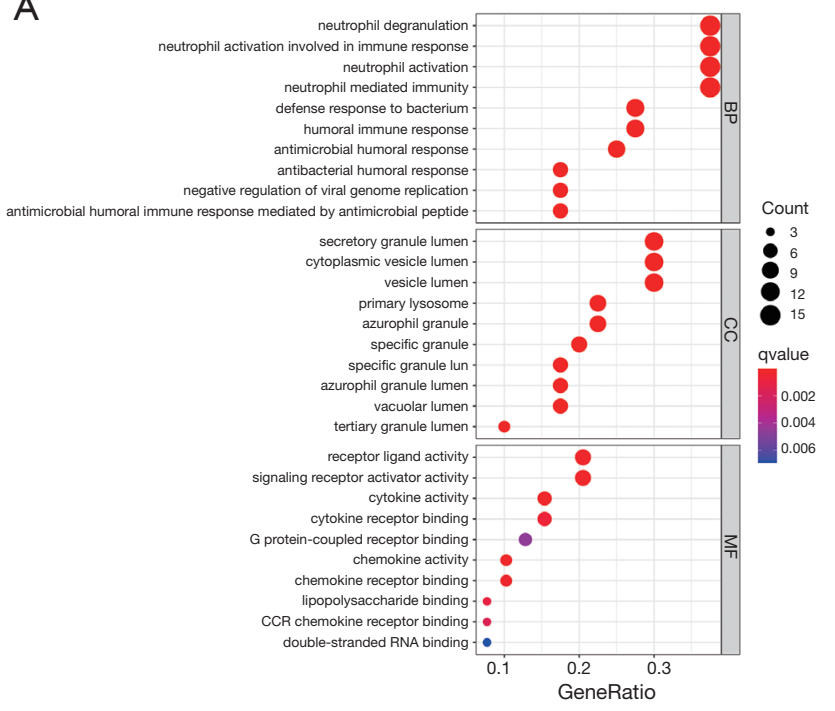

C

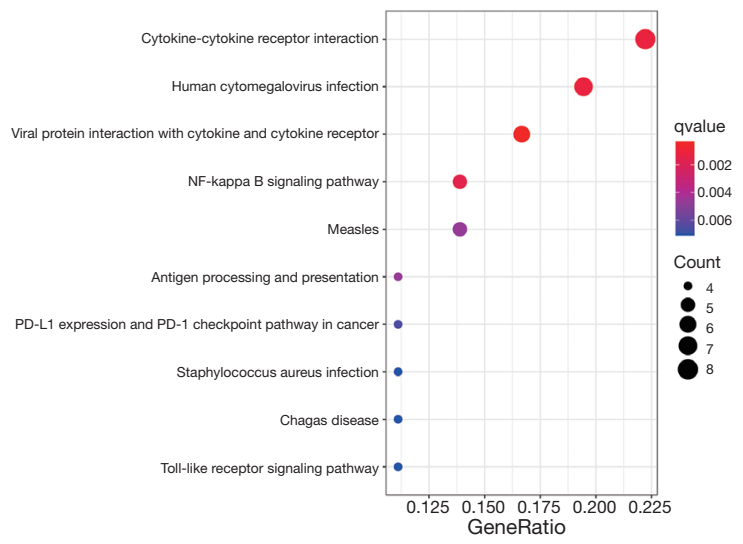

B
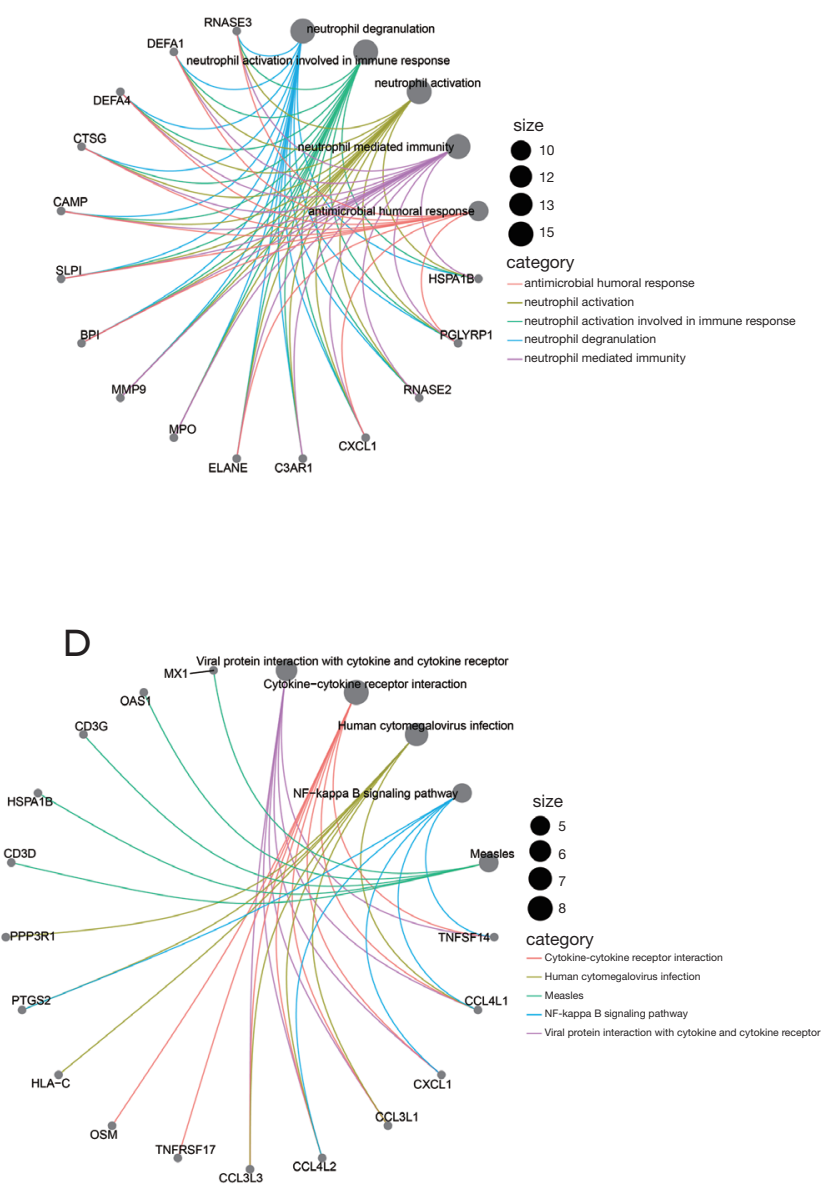

Figure 3 The enrichment functions of the immune related DEGs. The bubble diagram (A) and circus diagram (B) of GO enrichment functions. The bubble diagram (C) and circus diagram (D) of KEGG pathways. Red and blue dots represent q value, the radius size of dots represent the genes count. GO, Gene Ontology; KEGG, Kyoto Encyclopedia of Genes and Genomes; DEGs, differentially expressed genes.

(Figure 3C,D). From these findings, we can deduce that inflammation and immune response are important in the pathogenesis of epilepsy.

\section{Construction of the PPI network}

To explore the relation of immune related DEGs, we used the STRING database to construct a PPI network, and the top 10 interacted genes were used for further analysis. The results showed that the top 10 interacted genes included CXCL1, ELANE, MPO, BPI, CAMP, DEFA4, CTSG, $M M P 9, S L P I$, and HLA-C; among them, CXCL1 is the most interactive immune related DEG (Figure $4 A, B$ ). These results further confirmed that inflammation and immune response play important roles in the pathological process of epilepsy.

\section{Analysis of immune cell infiltration}

Further, we used the CIBERSORT algorithm to investigate the abundance of immune cell in individual samples (Figure $5 \mathrm{~A}$ ). The relationship results of various immune cells indicated that neutrophils inhibited gamma delta $\mathrm{T}$ cells, CD8 T cells, activated CD4 memory T cells, and monocytes, but positively regulated M0 macrophages; gamma delta $\mathrm{T}$ cells positively regulated T cells CD8 and 
A

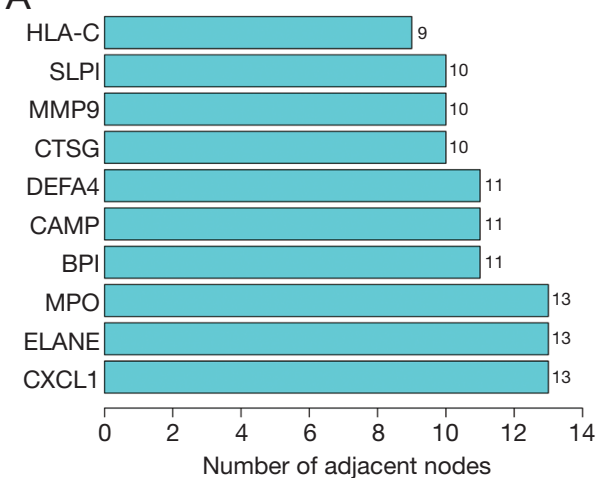

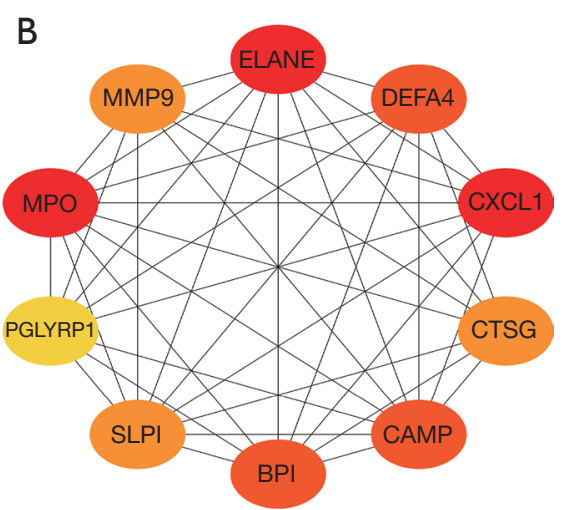

Figure 4 PPI network of immune related DEGs in epilepsy. Histogram of number of adjacent nodes of the top 10 genes in the PPI network (A). The protein-protein interaction network of the top 10 genes (B). The redder the color, the more adjacent nodes number of genes. PPI, protein-protein interaction; DEGs, differentially expressed genes.

A

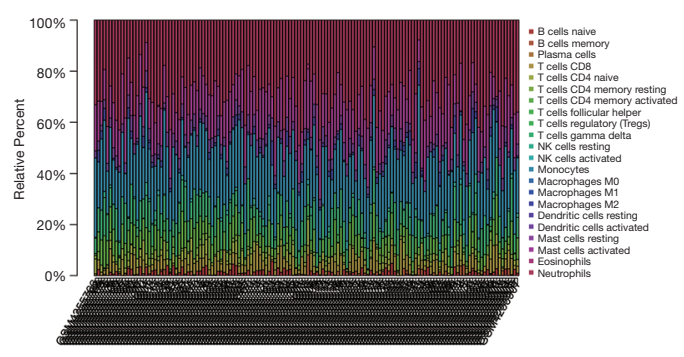

C

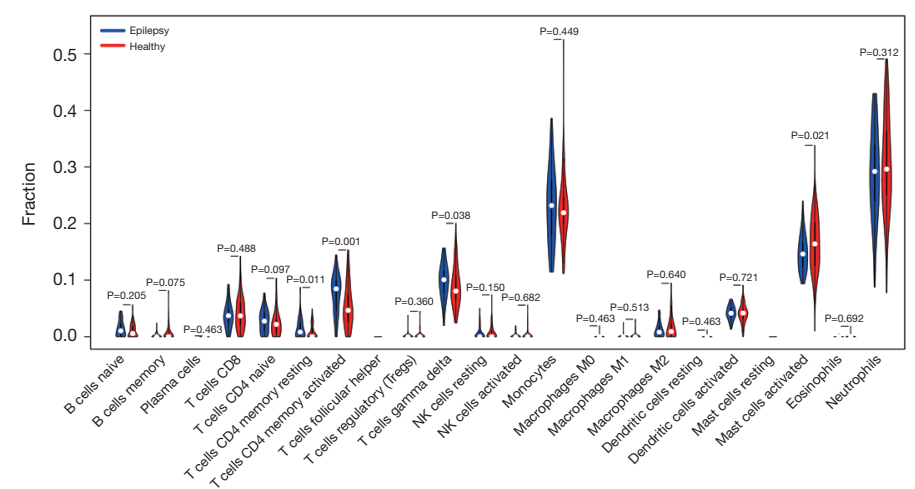

B

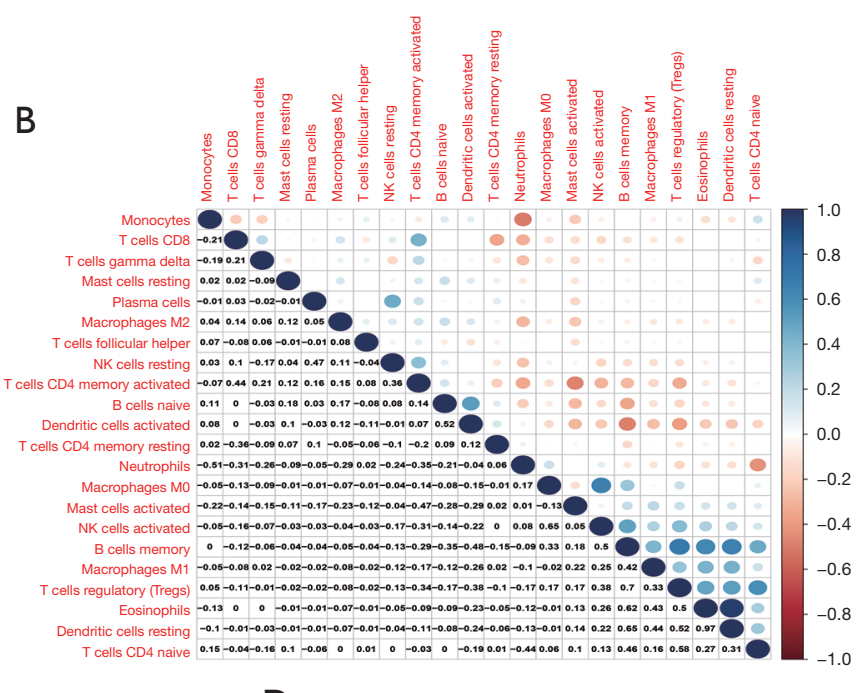

D 


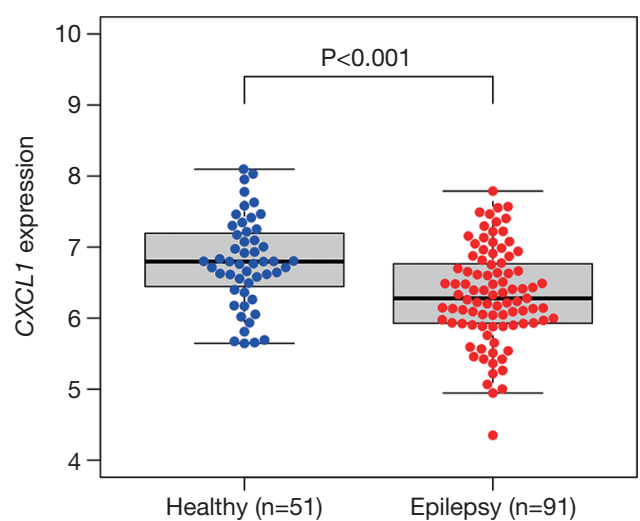

Figure 6 The expression level of CXCL1 between epilepsy and normal samples. CXCL1, C-X-C motif chemokine ligand 1.

activated CD4 memory $\mathrm{T}$ cells, but negatively regulated monocytes, resting natural killer (NK) cells, activated mast cells, neutrophils, and so on (Figure 5B). The results also showed that epilepsy patients with higher activated mast cells infiltration $(\mathrm{P}=0.021)$, but lower activated $\mathrm{CD} 4$ memory $\mathrm{T}$ cells $(\mathrm{P}=0.001)$, resting $\mathrm{CD} 4$ memory $\mathrm{T}$ cells $(\mathrm{P}=0.011)$, and gamma delta $\mathrm{T}$ cells $(\mathrm{P}=0.038)$ infiltration (Figure $5 C, D$ ). These results revealed the association of different immune cells.

\section{Expression level of CXCL1}

The gene CXCL1 was the hub gene in the PPI network, so we explored its expression level between the epilepsy and normal samples. The results showed that CXCL1 was lowly expressed in epilepsy patients $(\mathrm{P}<0.001)$ (Figure 6).

\section{The association between CXCL1 and immune cell infiltration}

The results of correlation analysis indicated that there was a positive correlation between CXCL1 and activated mast cells ( $\mathrm{R}=0.25, \mathrm{P}=0.019)$ and neutrophils $(\mathrm{R}=0.3, \mathrm{P}=0.0043)$, and a negative correlation with gamma delta $\mathrm{T}$ cells $(\mathrm{R}=-0.25$, $\mathrm{P}=0.018$ ) (Figure 7).

\section{Behavioral observation}

A total of 4 rats died during persistent generalized tonic clonic seizure (on days $5,9,18$, and 21 of continuous intraperitoneal injection of PTZ). A total of 17 PTZtreated rats developed consecutive seizures of 3 times of grade IV or above for 16-24 days. Additionally, 3 rats did not reach the ignition standard after 28 days of continuous intraperitoneal PTZ injection.

\section{Validation of CXCL1 messenger RNA expression}

Our results indicated the significant downregulation of CXCL1 messenger RNA (mRNA) in a chronic epilepsy model (control: 0.9898 \pm 0.07736 ; epilepsy: $0.7072 \pm 0.07319$, $\mathrm{P}<0.001$ ) (Figure 8).

\section{Discussion}

Through better understanding of the underlying mechanisms of epilepsy, it has become apparent that the immune system plays a significant role in the development of seizures. Vezzani et al. revealed the key role of cytokines in epilepsy (15). Further, activation of the innate immune system and inflammation can have a deep influence on the development of seizures (16). Based on these factors, we conducted this study to identify the significant differentially expressed IRGs between epilepsy and normal samples, and explored the biological functions and expression levels, further using PCR to validate the genes expression levels.

The raw data was downloaded from GSE143272; among a total of 245 DEGs, there were 143 upregulated and 102 downregulated genes in epilepsy, and there were 1,811 IRGs in the ImmPort database. Finally, we obtained 44 differential IRGs via intersection of DEGs and IRGs. The enrichment function results showed that these DEGs are involved in different immune response processes such as neutrophil degranulation, neutrophil activation involved in immune response, neutrophil mediated immunity, neutrophil activation, chemokine receptor binding, chemokine activity, MHC protein complex binding, viral protein interaction with cytokine and cytokine receptor, and cytokine-cytokine receptor interaction. We then selected IRGs via the PPI network, and detected 10 genes, including CXCL1, ELANE, MPO, BPI, CAMP, DEFA4, CTSG, MMP9, SLPI, and $H L A-C$, and the CXCL1 gene was selected for further analysis. Finally, we explored the immune cells abundance of individual samples and the relationship between CXCL1 and immune cell infiltrations.

Over the past decades, the inflammatory and immune responses have been suspected of participating in one of the potential underlying mechanisms for epilepsy $(17,18)$, and intravenous immune globulin has subsequently been used in the treatment of intractable childhood epilepsy. Immune 
A

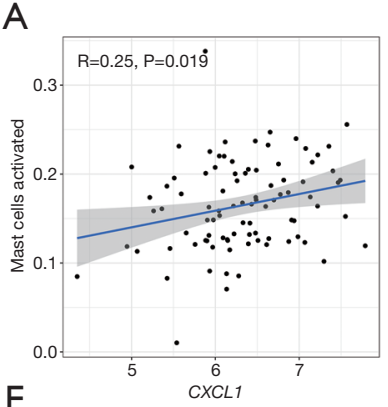

E
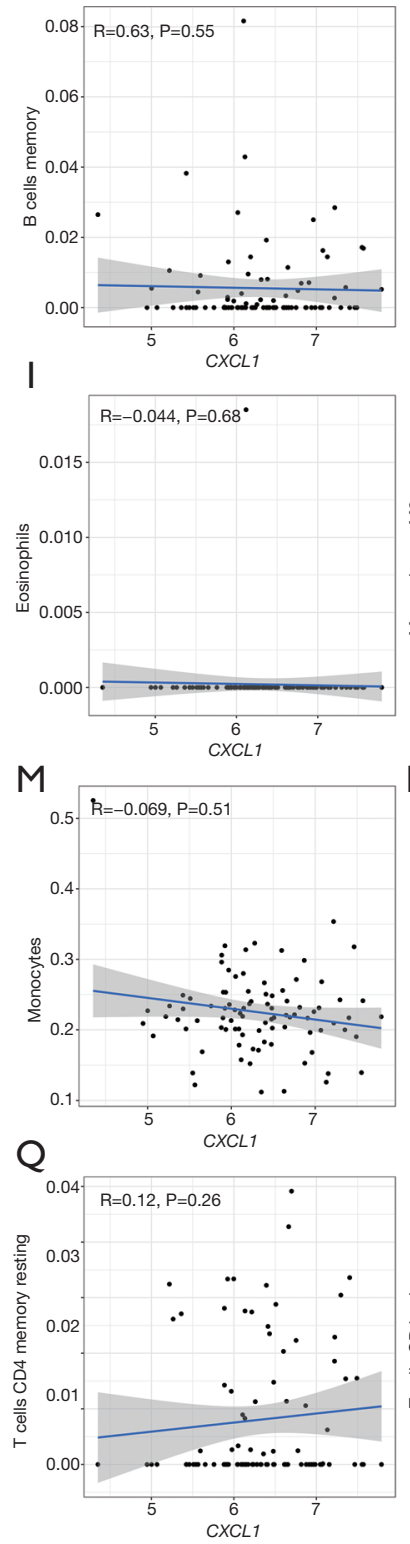

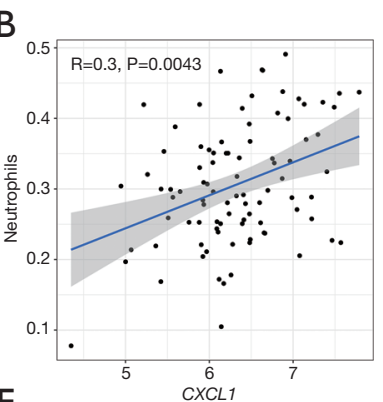

B

F
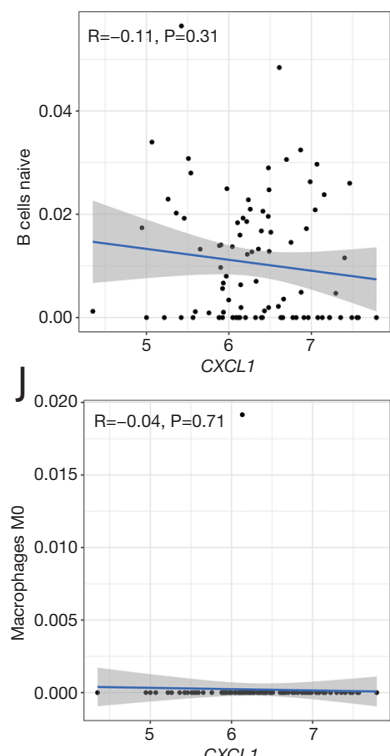

N
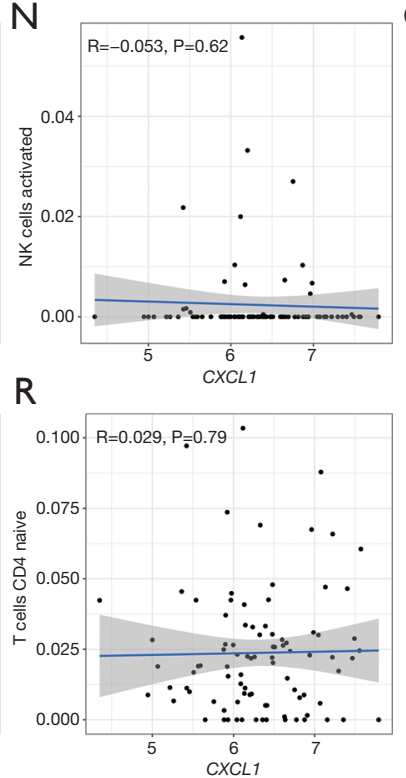

C

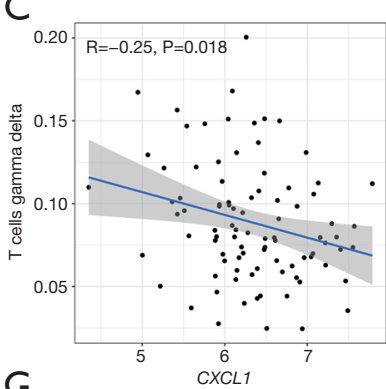

G
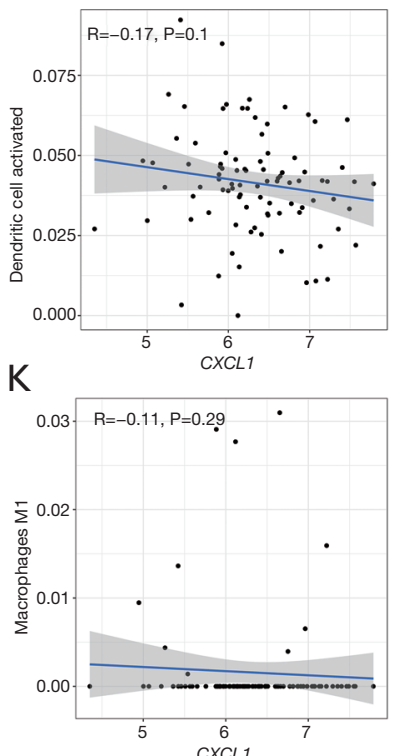

$\mathrm{O}$
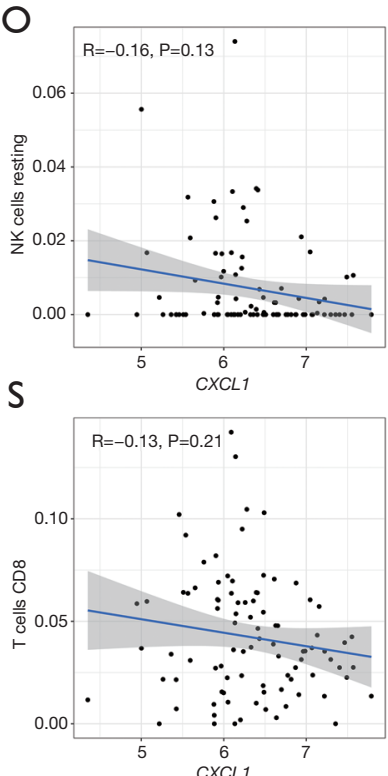

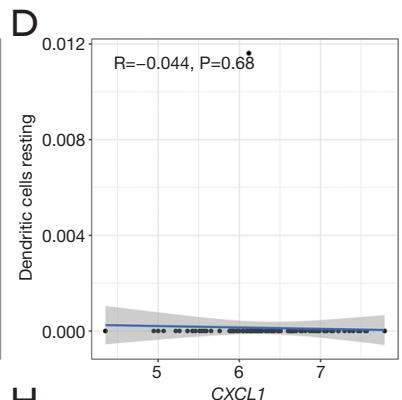

$\mathrm{H}$

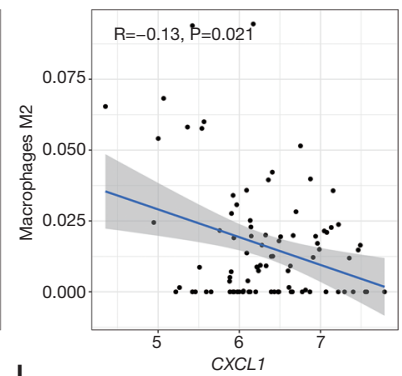

L
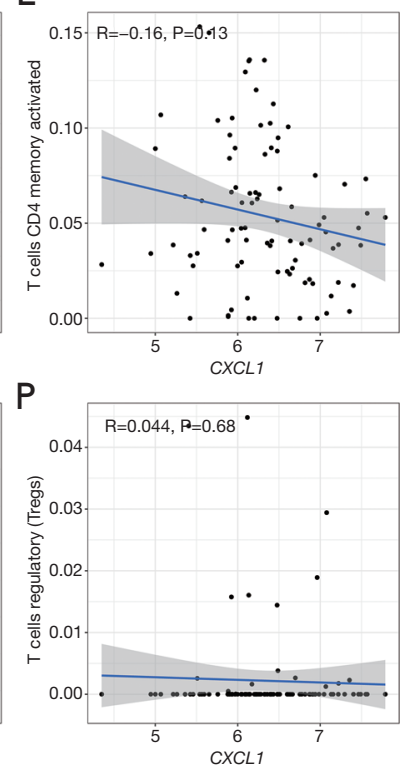

Figure 7 Relationship between CXCL1 and immune cells in epilepsy. Activated mast cells (A) and neutrophils (B), gamma delta T cells (C), resting dendritic cells (D), memory B cells (E), naïve B cells (F), activated dendritic cells (G), M2 macrophages (H), eosinophils (I), M0 macrophages (J), M1 macrophages (K), activated CD4 memory T cells $(\mathrm{L})$, monocytes $(\mathrm{M})$, activated NK cells $(\mathrm{N})$, resting NK cells $(\mathrm{O})$, regulatory T cells (P), resting CD4 memory T cells (Q), naïve CD4 T cells (R), CD8 T cells (S). CXCL1, C-X-C motif chemokine ligand 1. 


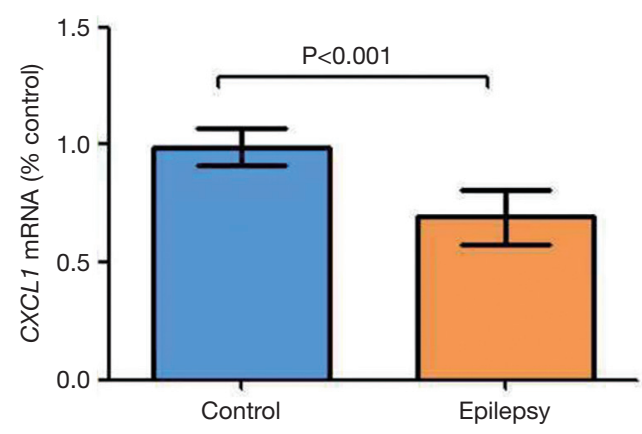

Figure 8 The validation of mRNA transcriptional levels via rat models. mRNA, messenger RNA.

cells are one of main components of the immune system, and IRGs may influence immune cell infiltration and signaling pathway transduction to affect the development of seizures. Regrettably, few studies have investigated the association between immune cells and epilepsy. The MMP9 gene has been shown to promote inflammation and the development of seizures (19-22), and a relationship has been identified between $H L A$ and epilepsy (23-26). Furthermore, CXCL1 has an association with epilepsy (27); however, the actual roles of $H L A-C$ and $C X C L 1$ in epilepsy have remained obscure. Therefore, the relationship between these IRGs and epilepsy has required further investigation. Via the regulation of immune cells infiltration, IRGs may significantly influence the pathophysiology of epilepsy. Herein, we investigated the difference between immune cells infiltration of epilepsy samples and normal samples. The results showed that epilepsy patients with higher activated mast cells, but lower activated CD4 memory $\mathrm{T}$ cells, resting CD4 memory $\mathrm{T}$ cells, and gamma delta $\mathrm{T}$ cells infiltration. Kilinc et al. indicated that mast cells may ameliorate the development of seizures (28); however, there have been few studies investigating the role of $\mathrm{T}$ cells in epilepsy. Since only CXCL1, MMP9, and HLA-C have been shown to have relationships with epilepsy, we explored the expression levels of these genes and their association with immune cells infiltration. The results showed that epilepsy patients had significantly higher expression levels of $H L A-C$ and $M M P 9$. It was revealed that $H L A-C$ was positively correlated with neutrophils but negatively correlated with naive CD4 $\mathrm{T}$ cells. It was also shown that MMP9 was positively correlated with neutrophils, activated mast cells, M2 macrophages infiltration, but inhibited CD8 T cells, naïve CD4 T cells, activated CD4 memory T cells, monocytes, activated dendritic cells, and naïve $\mathrm{B}$ cells infiltration. Based on these associations, IRGs may have a profound influence on epilepsy via regulation of immune cell infiltration and regulating inflammation. However, the role of these immune cells in epilepsy is still unclear. Thus, further investigation of the underlying mechanism of these immune cells in epilepsy urgently needed, which may facilitate finding a novel treatment or supplementary therapy regimens for epilepsy patients.

\section{Conclusions}

In present study, the results showed that IRGs such as CXCL1, MMP9, and HLA-C have a significant influence on epilepsy, and they may work via regulating immune cells infiltration. However, the underlying mechanism of some IRGs and immune cells for epilepsy has yet to be elucidated.

\section{Acknowledgments}

Funding: None.

\section{Footnote}

Reporting Checklist: The authors have completed the ARRIVE reporting checklist. Available at https://dx.doi. org/10.21037/atm-21-2792

Data Sharing Statement: Available at https://dx.doi. org/10.21037/atm-21-2792

Conflicts of Interest: All authors have completed the ICMJE uniform disclosure form (available at https://dx.doi. org/10.21037/atm-21-2792). The authors have no conflicts of interest to declare.

Ethical Statement: The authors are accountable for all aspects of the work in ensuring that questions related to the accuracy or integrity of any part of the work are appropriately investigated and resolved. The study was conducted in accordance with the Declaration of Helsinki (as revised in 2013). Experiments were performed under a project license (No.: 202101003) granted by The First Affiliated Hospital of Guangxi Medical University ethics board, in compliance with Chinese guidelines for the care and use of animals.

Open Access Statement: This is an Open Access article distributed in accordance with the Creative Commons 
Attribution-NonCommercial-NoDerivs 4.0 International License (CC BY-NC-ND 4.0), which permits the noncommercial replication and distribution of the article with the strict proviso that no changes or edits are made and the original work is properly cited (including links to both the formal publication through the relevant DOI and the license). See: https://creativecommons.org/licenses/by-nc-nd/4.0/.

\section{References}

1. Thijs RD, Surges R, O'Brien TJ, et al. Epilepsy in adults. Lancet 2019;393:689-701.

2. Wang D, Wei P, Shan Y, et al. Optimized stereoelectroencephalography-guided radiofrequency thermocoagulation in the treatment of patients with focal epilepsy. Ann Transl Med 2020;8:15.

3. Pitkänen A, Sutula TP. Is epilepsy a progressive disorder? Prospects for new therapeutic approaches in temporal-lobe epilepsy. Lancet Neurol 2002;1:173-81.

4. Choi J, Nordli DR Jr, Alden TD, et al. Cellular injury and neuroinflammation in children with chronic intractable epilepsy. J Neuroinflammation 2009;6:38.

5. Riazi K, Galic MA, Pittman QJ. Contributions of peripheral inflammation to seizure susceptibility: cytokines and brain excitability. Epilepsy Res 2010;89:34-42.

6. Wheless JW, Clarke DF, Arzimanoglou A, et al. Treatment of pediatric epilepsy: European expert opinion, 2007. Epileptic Disord 2007;9:353-412.

7. Dinarello CA. Infection, fever, and exogenous and endogenous pyrogens: some concepts have changed. J Endotoxin Res 2004;10:201-22.

8. RASMUSSEN T, OLSZEWSKI J, LLOYDSMITH D. Focal seizures due to chronic localized encephalitis. Neurology 1958;8:435-45.

9. Vincent A, Bien CG. Anti-NMDA-receptor encephalitis: a cause of psychiatric, seizure, and movement disorders in young adults. Lancet Neurol 2008;7:1074-5.

10. Dalmau J, Gleichman AJ, Hughes EG, et al. Anti-NMDAreceptor encephalitis: case series and analysis of the effects of antibodies. Lancet Neurol 2008;7:1091-8.

11. Tchekalarova J, Sotiriou E, Angelatou F. Down-regulation of dopamine D1 and D2 receptors in the basal ganglia of PTZ kindling model of epilepsy: effects of angiotensin IV. Brain Res 2004;1024:159-66.

12. Hunot S, Vila M, Teismann P, et al. JNK-mediated induction of cyclooxygenase 2 is required for neurodegeneration in a mouse model of Parkinson's disease. Proc Natl Acad Sci U S A 2004;101:665-70.
13. Racine RJ. Modification of seizure activity by electrical stimulation. II. Motor seizure. Electroencephalogr Clin Neurophysiol 1972;32:281-94.

14. Lin W, Qin J, Ni G, et al. Downregulation of hyperpolarization-activated cyclic nucleotide-gated channels (HCN) in the hippocampus of patients with medial temporal lobe epilepsy and hippocampal sclerosis (MTLE-HS). Hippocampus 2020;30:1112-26.

15. Vezzani A, French J, Bartfai T, et al. The role of inflammation in epilepsy. Nat Rev Neurol 2011;7:31-40.

16. Vezzani A, Granata T. Brain inflammation in epilepsy: experimental and clinical evidence. Epilepsia 2005;46:1724-43.

17. Aronica E, Ravizza T, Zurolo E, et al. Astrocyte immune responses in epilepsy. Glia 2012;60:1258-68.

18. Walker L, Sills GJ. Inflammation and epilepsy: the foundations for a new therapeutic approach in epilepsy? Epilepsy Curr 2012;12:8-12.

19. Cai X, Long L, Zeng C, et al. LncRNA ILF3-AS1 mediated the occurrence of epilepsy through suppressing hippocampal miR-212 expression. Aging (Albany NY) 2020;12:8413-22.

20. Cudna A, Jopowicz A, Mierzejewski P, et al. Serum metalloproteinase 9 levels increase after generalized tonicclonic seizures. Epilepsy Res 2017;129:33-6.

21. Nass RD, Wagner M, Surges R, et al. Time courses of HMGB1 and other inflammatory markers after generalized convulsive seizures. Epilepsy Res 2020;162:106301.

22. Rüber T, David B, Lüchters G, et al. Evidence for periictal blood-brain barrier dysfunction in patients with epilepsy. Brain 2018;141:2952-65.

23. Hu FY, Wang W, Ren JC, et al. Levetiracetam-induced cutaneous adverse drug reactions were not associated with HLA genes in a small sample of Chinese patients with epilepsy. Epilepsy Res 2016;124:12-5.

24. Iannetti P, Morellini M, Raucci U, et al. HLA antigens, epilepsy and cytomegalovirus infection. Brain Dev 1988;10:256-8.

25. Jain S, Padma MV, Kanga U, et al. Family studies and human leukocyte antigen class II typing in Indian probands with seizures in association with single small enhancing computed tomography lesions. Epilepsia 1999;40:232-8.

26. Zhao T, Li HJ, Wang TT, et al. Association between HLA genotype and antiseizure medications (ASMs)-induced maculopapular eruption among epilepsy patients in Xinjiang, China. Epilepsy Res 2020;165:106391. 
27. Liu XX, Yang L, Shao LX, et al. Endothelial Cdk5 deficit leads to the development of spontaneous epilepsy through CXCL1/CXCR2-mediated reactive astrogliosis. J Exp Med 2020;217:e20180992.

28. Kilinc E, Torun IE, Cetinkaya A, et al. Mast cell activation

Cite this article as: Luo X, Xiang T, Huang H, Ye L, Huang Y, $\mathrm{Wu}$ Y. Identification of significant immune-related genes for epilepsy via bioinformatics analysis. Ann Transl Med 2021;9(14):1161. doi: 10.21037/atm-21-2792 ameliorates pentylenetetrazole-induced seizures in rats: The potential role for serotonin. Eur J Neurosci 2021. [Epub ahead of print]. doi: 10.1111/ejn.15145.

(English Language Editor: J. Jones) 